\title{
Ceratoplastia lamelar em cães usando membrana amniótica equina. Estudo clínico e morfológico
}

\author{
Lamelar keratoplasty in dogs using equine amniotic membrane. \\ Clinical and morphological study
}

\author{
Andréa BARBOSA ${ }^{1}$; Paulo Sergio de Moraes BARROS ${ }^{2}$; José Luiz GUERRA ${ }^{3}$; Denise Aya OTSUKI ${ }^{4}$ \\ ${ }^{1}$ Medicina Veterinária da Universidade de Santo Amaro, São Paulo - SP, Brasil \\ ${ }^{2}$ Faculdade de Medicina Veterinária e Zootecnia da Universidade de São Paulo, São Paulo - SP, Brasil \\ ${ }^{3}$ Medicina Veterinária da Universidade Anhembi-Morumbi, São Paulo - SP, Brasil \\ ${ }^{4}$ Faculdade de Medicina da Universidade de São Paulo, São Paulo - SP, Brasil
}

\begin{abstract}
Resumo
A membrana amniótica (MA) consolidou-se no tratamento de afecções na superfície ocular. O objetivo deste estudo foi avaliar a viabilidade do implante de MA equina em ceratoplastia lamelar de cães. As membranas amnióticas foram preservadas em glicerina (98\%). A eficácia do implante foi acompanhada por avaliação clínica, tempo de cicatrização, resposta inflamatória e reconstrução da arquitetura da córnea. Foram selecionados 12 cães, que foram divididos em quatro grupos de três animais. Em cada animal, foi realizada ceratotomia lamelar com 5 mm de diâmetro, seguida de aplicação do implante de MA. Após cirurgia, os animais foram avaliados em diferentes tempos: 2, 7, 21 e 40 dias. Durante o período de observação, os exames oftalmológicos foram realizados com intervalo de 48 h e, após a última avaliação, os animais foram submetidos à eutanásia. Os olhos foram enucleados, fixados e corados com hematoxilina-eosina (HE), ácido periódico de Schiff (PAS) e picrossirius. Os implantes foram completamente epitelizados em cerca de 10 dias após a cirurgia. Os neovasos apresentaram involução progressiva a partir de 21 dias e não foram detectados ao final de 40 dias pós-cirurgia, restando apenas uma nébula no local da lesão. À microscopia óptica, observou-se resposta inflamatória moderada, presença de epitélio pavimentoso estratificado aos sete dias e epitelização completa aos 21 dias. Aos 40 dias, a membrana basal do epitélio apresentou-se reconstituída. Assim, concluímos que a membrana amniótica equina é viável como implante em córnea de cão, sendo incorporada ao estroma e resultando em restabelecimento parcial da transparência.
\end{abstract}

Palavras-chave: Córnea. Cães. Membrana amniótica. Oftalmologia veterinária.

\begin{abstract}
Amniotic membranes have been successfully used in the treatment of superficial corneal diseases. The purpose of this study was to assess the feasibility of use of equine amniotic membrane (AM) in lamellar keratoplasty of dogs. Amniotic membranes were preserved in glycerol (98\%). Implant efficacy was assessed by clinical evaluation, time of healing, inflammatory response, and the corneal architectural configuration. Twelve dogs were divided into four groups of three animals. In each animal, a 5-mm diameter lamellar keratectomy was performed, and followed by implantation of the AM. The animals were evaluated at the $2^{\text {th }}, 7^{\text {th }}, 21^{\text {th }}$, and $40^{\text {th }}$ days after surgery. During this period, ophthalmological exams were performed at 48-h intervals, and the animals were euthanized after the last evaluation. The eyes were enucleated, included, fixed, and stained with hematoxylin-eosin (HE), periodic acid-Schiff (PAS), and picrosirius. Epithelization of the implants was completed in about ten days after surgery. New vessels progressively diminished from 21 days on, were not detected on day 40 after surgery, and only a nebula was observed in the implant area. Optical microscopy revealed a mild inflammatory response, presence of squamous epithelium on day seven, and total epithelization on day 21 after surgery. On day 40, the basal epithelial membrane was shown to be reformed. Therefore, we conclude that use of equine amniotic membranes is feasible as implant for dog cornea since it is incorporated to the corneal stroma, and a relative transparency can be obtained.
\end{abstract}

Keywords: Cornea. Dogs. Amniotic membrane. Veterinary ophthalmology.

Correspondência para:

Andréa Barbosa

Av. Interlagos, 871 , bloco 7, apto. 24

04661-100, Jd. Umuarama, São Paulo, SP e-mail: barbosa.a@uol.com.br

Recebido: 17/01/2013

Aprovado: 03/07/2013 


\section{Introdução}

O transplante de membrana amniótica (MA) para tratamento de ceratites ulcerativas é objeto de extensa pesquisa em oftalmologia humana e veterinária, devido ao seu potencial na reconstrução da superfície ocular. A aplicação da MA beneficia o processo de epitelização, facilitando a adesão e migração das células epiteliais basais, prevenindo a apoptose e restaurando o fenótipo epitelial (GOMES et al., 1999; TSENG; PRABHASAWAT; LEE, 1997; AZUARA-BLANCO; PILLAI; DUA, 1999; PIRES; PIRES; TSENG, 1999; MELLER et al., 2000), além de reduzir os processos inflamatório, angiogênico e cicatricial (TSENG et al., 1998), tem atividade antimicrobiana comprovada e é imunologicamente inerte (GOMES et al., 1999; SHIMAZAKI; YANG; TSUBOTA, 1997; KUBO et al., 2001). O transplante pode, ainda, promover a proliferação e a diferenciação de células germinativas do limbo e da conjuntiva (MELLER et al., 2000). A membrana fetal é formada por córion, alantoide $\mathrm{e}$ amnion, sendo a MA a parte mais interna em contato com o feto (BARROS et al., 2005).

O primeiro relato de uso de membrana amniótica, em oftalmologia, foi de De Rötth (1940) na reparação de simbléfaro e defeitos conjuntivais. Em medicina veterinária, a MA foi utilizada para implante em lesões de córnea em cães, inicialmente por Barros et al. (1998) que aplicaram a membrana xenógena (equina) em ceratectomias penetrantes, em cães. A seguir, Godoy, Guerra e Barros (2002) avaliaram a viabilidade da membrana fetal equina para ceratoplastia lamelar, com resposta inflamatória menor do que a descrita em implantes similares de outras membranas biológicas (GARCIA et al., 1996; ANDRADE et al., 1999; TEIXEIRA; BARROS; MARTINS, 2004). Outros estudos experimentais com implante de MA foram descritos por Safatle et al. (2002); Andrade (2003); Souza (2003) e Milani et al. (2008), confirmando os benefícios na cicatrização da superfície ocular.

O objetivo deste trabalho foi avaliar a viabilidade e a eficácia do implante de MA equina, preservada em glicerina a 98\%, na reparação de ceratectomias lamelares, em cães, por avaliações clínica pós-operatória dos animais, do tempo de cicatrização, da reconstrução da arquitetura da córnea e da resposta inflamatória.

\section{Material e Métodos}

Foram formados quatro grupos de três cães, machos ou fêmeas, sem raça definida, nos quais foi implantada MA na córnea, após a ceratectomia lamelar. Optou-se pela realização do procedimento em um dos olhos de cada animal, direito ou esquerdo, aleatoriamente. Os grupos tiveram tempos de observação distintos: 48 horas (G I), 7 (G II), 21 (G III) e 40 dias (G IV), após os quais, os olhos que receberam os implantes foram enucleados e fixados para posterior análise. Os animais receberam os cuidados básicos de saúde e higiene, seguindo as normas para experimentação oftálmica da "Association for Research in Vision and Ophthalmology" (ARVO).

A MA foi coletada de forma asséptica, logo após o parto de égua clinicamente sadia, teve sua face epitelial identificada pela colocação de um ponto de fio de nylon antes de separá-la do feto recém-nascido. Ato contínuo, a MA foi submetida à lavagem com solução fisiológica a $0,9 \%$ e imersa em solução de glicerina $98 \%$. Antes da cirurgia, os fragmentos foram recortados no tamanho adequado e mantidos em solução fisiológica 0,9\% para hidratação por, no mínimo, 15 minutos. $\mathrm{O}$ protocolo anestésico estabelecido foi sedação com acepromazina (Acepran 0,2\% - Univet) $(0,05 \mathrm{mg} / \mathrm{kg} / \mathrm{IM}) \mathrm{e}$ meperidina (Dolosal - Cristália) $(2 \mathrm{mg} / \mathrm{kg}, \mathrm{IM})$, indução com propofol (Propovan - Cristália) (5 mg / kg, IV) e, para manutenção, utilizou-se isofluorano (Isoforine - Cristália) e bloqueador neuromuscular rocurônio (Esmeron - Akzo Nobel Ltda.) (0,6 mg/kg, IV). Para analgesia pré e pós-operatória, foi usado flunixin meglumine (Banamine - Schering Plough) (1 mg/kg, SC) e no pós-operatório imediato, cloridrato de tramadol (2 mg/kg, I.V. ) (Tramadon - Cristália).

Sob microscópio cirúrgico com aumento de 10 vezes procedeu-se a ceratectomia lamelar, com profundidade 
de $0,3 \mathrm{~mm}$ e $5 \mathrm{~mm}$ de diâmetro, com auxílio de trépano de Castroviejo ( $5 \mathrm{~mm}$ ) e uma lâmina de bisturi número 15 , na região temporal, a 2 milímetros do limbo. Os retalhos de MA de $6 \mathrm{~mm}$ de diâmetro foram suturados nos bordos da lesão de córnea, em pontos simples separados não penetrantes, com fio de náilon 8.0 monofilamentado, agulha trifacetada $0,65 \mathrm{~cm}$, sendo a MA aplicada com sua face mesenquimal voltada para a córnea (Figura 2). Após o procedimento, os animais foram mantidos com colar elisabetano e receberam colírio de Tobramicina $0,3 \%$ (Tobramicina $0,3 \%$ - Alcon), a cada doze horas, nos primeiros sete dias.

No período pós-operatório, os cães foram submetidos, em dias alternados, ao exame clínico do olho com auxílio de lupa e fonte luminosa, sendo avaliados quanto à presença de blefarospasmo, fotofobia, secreção ocular, permanência dos implantes, neoformação vascular e edema corneais. Foram realizados os testes lacrimal de Schirmer e de fluoresceína em todas as avaliações. Os parâmetros foram classificados de forma subjetiva em: 0 (ausente), 1 (leve), 2 (moderado) e 3 (acentuado). Os pontos de sutura foram retirados após a completa epitelização do implante, confirmada pelo teste de fluoresceína com resultado negativo, entre nove e quinze dias de pós-operatório, para evitar estímulo adicional à resposta inflamatória, sendo o procedimento realizado sob anestesia endovenosa com propofol. Ao final do período de observação prédeterminado, os animais foram submetidos à eutanásia, por injeção intravenosa de tiopental sódico (Tiopental - Cristália) para anestesia em plano profundo e, em seguida, de cloreto de potássio 19,1\%. Os olhos em estudo foram então enucleados pela técnica transconjuntival descrita por Slatter (2001) e mergulhados em solução de formol 10\% tamponado. Após a fixação, os segmentos córneo-esclerais foram seccionados e incluídos em blocos de parafina. Cortes de $5 \mu \mathrm{m}$ de espessura, obtidos com micrótomo, foram corados por hematoxilina-eosina (HE), ácido periódico de Schiff (PAS) e Picrossirius e analisados sob microscopia óptica de luz.

\section{Resultados}

Blefarospasmo e hiperemia conjuntival foram observados nos animais de todos os grupos, sendo mais intensos nos quatro primeiros dias, depois, apresentaram redução gradativa até desapareceram após a retirada das suturas. Fotofobia leve a moderada foi observada nos quatro primeiros dias de pós-operatório, em todos os grupos, exceto em um cão do G IV, que apresentou esta alteração clínica até o nono dia. A secreção ocular presente nos cães de todos os grupos foi predominantemente mucosa, com quantidade variável entre os graus 1 e 3 , sendo a sua redução progressiva e mais acentuada após a retirada das suturas. Quemose moderada foi observada nos primeiros dois a quatro dias, em todos os animais (Figura 1). Edema de córnea manifestou-se em sete cães (distribuídos em todos os grupos), sendo restrito à periferia do implante. O teste de fluoresceína foi usado na determinação do período necessário para completa epitelização do implante, que aconteceu em dez $( \pm 2)$ dias em dez animais; em um animal, o implante estava totalmente epitelizado no quarto dia e, em outro, somente no dia 16 (ambos do G III). Os primeiros neovasos foram observados no sexto dia, em cinco animais, no décimo dia, em três, e no dia 12 , em um animal. Os vasos atingiram a região do implante, entre os dias 8 e 12, em cinco dos seis animais observados (G III e G IV) (Figuras 2 e 3); em um cão os vasos não atingiram o implante. Nos animais do G IV, houve regressão da vascularização a partir do vigésimo dia e, aos 40 dias, os vasos não foram mais observados, restando apenas uma nébula no local do implante (Figura 4).

À microscopia óptica de luz, aos dois dias após o implante, a membrana foi visibilizada na superfície do estroma exposto pela ceratectomia; o tecido implantado apresentou-se amorfo, com baixa celularidade e eosinofílico (Figura 5). Foram encontrados raros fibroblastos e polimorfonucleares invadindo o tecido implantado. Observou-se início de epitelização, com formação de epitélio plano simples e maior proliferação celular nos bordos da lesão. Aos sete dias, havia 


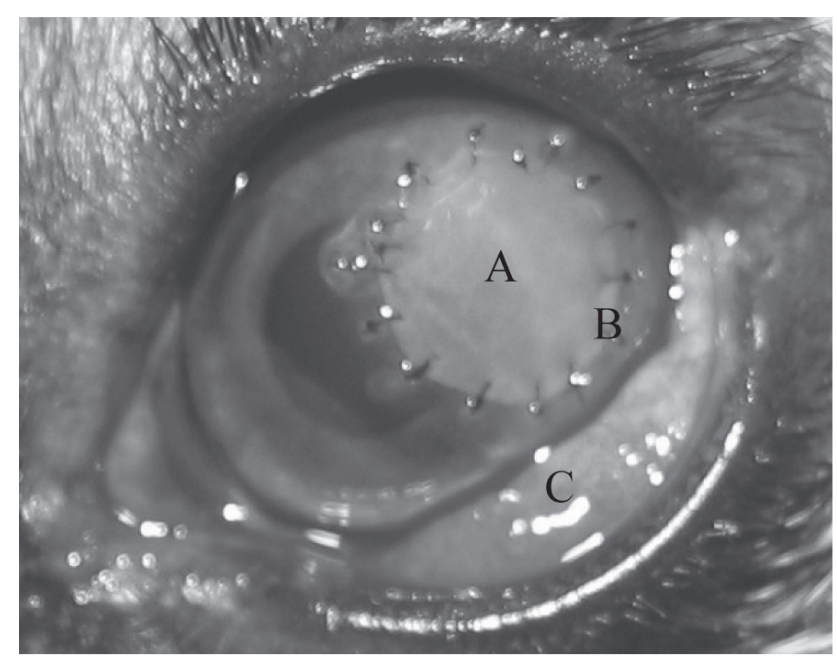

Figura 1 - Imagem fotográfica da córnea de cão aos dois dias após a ceratectomia lamelar e implante de MA equina

Nota: observa-se presença da MA (A), dos pontos de sutura (B), de congestão conjuntival e quemose $(C)$.

Fonte: (arquivos pessoais).

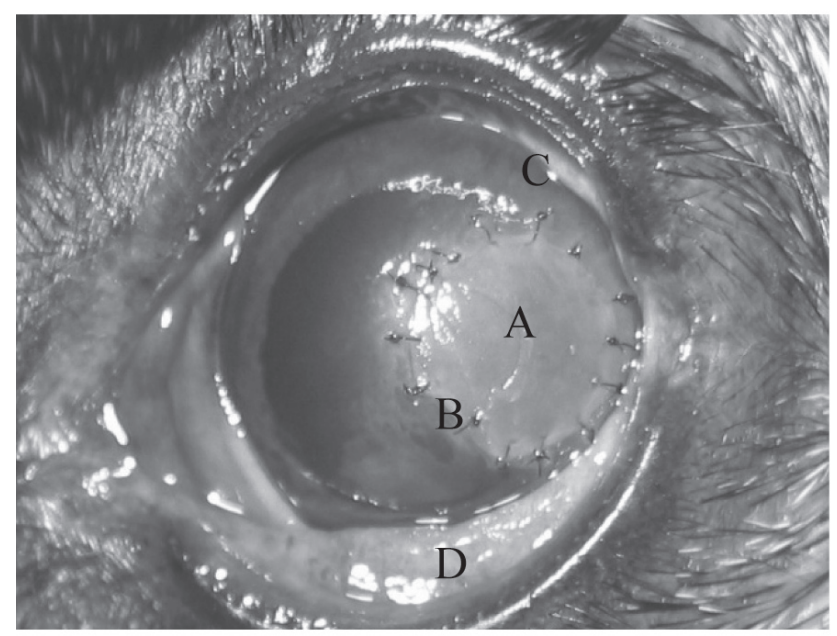

Figura 2 - Imagem fotográfica de olho de cão aos de sete dias após a ceratectomia lamelar e implante de MA equina

Nota: observa-se presença do implante de MA na córnea (A), pontos de sutura (B), formação da neovascularização periférica a partir do limbo (C), congestão conjuntival (D).

Fonte: (arquivos pessoais).



Figura 3 - Imagem fotográfica de olho de cão aos 15 dias de pós-operatório

Nota: observa-se implante totalmente incorporado ao tecido (A), neovasos invadindo terço lateral do implante (B); pontos de sutura já foram retirados.

Fonte: (arquivos pessoais).

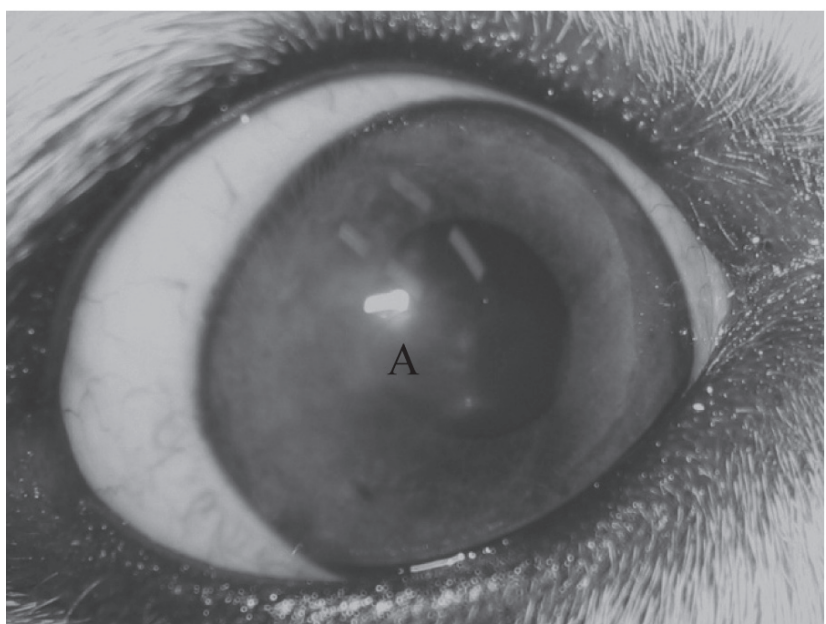

Figura 4 - Imagem fotográfica de olho de cão aos 40 dias após a ceratectomia lamelar e implante de MA equina

Nota: observa-se apenas nébula residual na região do implante $(A)$.

Fonte: (arquivos pessoais). 


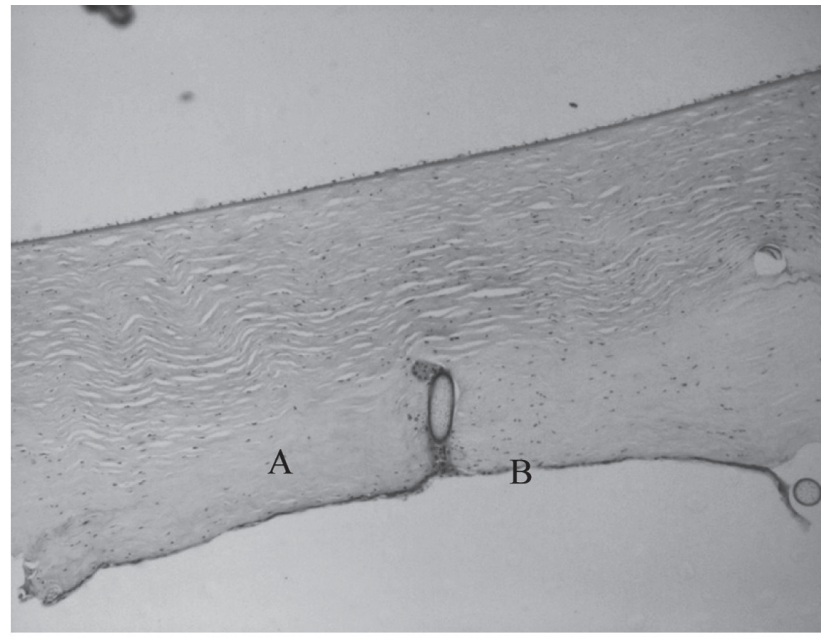

Figura 5 - Fotomicrografia de corte histológico de córnea com implante de MA equina, com dois dias de evolução pós-operatória, mostrando interface do tecido implantado com estroma normal da córnea.

Nota: o tecido implantado apresenta-se amorfo, com baixa celularidade e eosinófilo (A). Notase, ainda, formação de epitélio pavimentoso simples na superfície do tecido implantado (primórdios da epitelização) (B). Coloração: hematoxilina-eosina. Aumento: 40x.

Fonte: (arquivos pessoais). epitelização do implante com formação de epitélio pavimentoso estratificado, proliferação fibroblástica no interior do tecido implantado e discreto infiltrado inflamatório com polimorfonucleares, sendo possível observar, ainda nesta fase, a extensão do tecido implantado (Figura 6). Aos 21 dias, a epitelização do implante estava completa e a região de reparação apresentava maior número de camadas celulares, com perda da arquitetura epitelial inicial. Nessa fase, células inflamatórias não estavam presentes, mas havia presença de fibrócitos e fibroblastos. A angiogênese foi evidente nesse período. O tecido implantado apresentou-se incorporado à córnea, no entanto, observou-se que nesta área as fibras colágenas estromais estavam mais densamente distribuídas (Figura 7). Ao PAS, não foi possível identificar a membrana basal do epitélio. Aos 40 dias de pós-operatório, houve completa integração e epitelização do implante e a arquitetura celular do epitélio ainda não se apresentava completamente restabelecida. A estrutura colágena apresentou-se mais

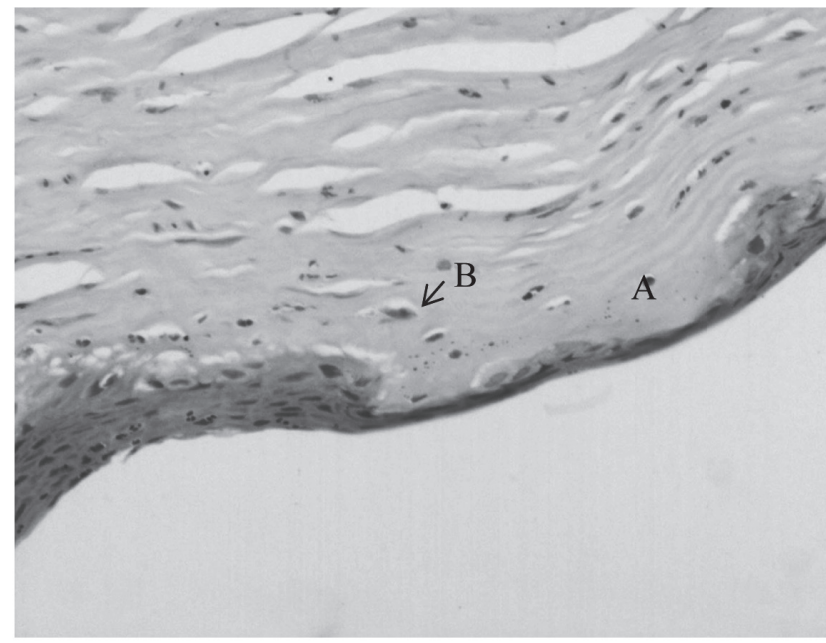

Figura 6 - Fotomicrografia de corte histológico de córnea com implante de MA equina, com sete dias de evolução pós-operatória, mostrando a extensão do tecido implantado com a superfície externa epitelizada $(\mathrm{A})$.

Nota: observa-se a proliferação fibroblástica no tecido implantado (B). Coloração: hematoxilina-osina. Aumento: $200 \mathrm{e} \mathrm{x}$.

Fonte: (arquivos pessoais).

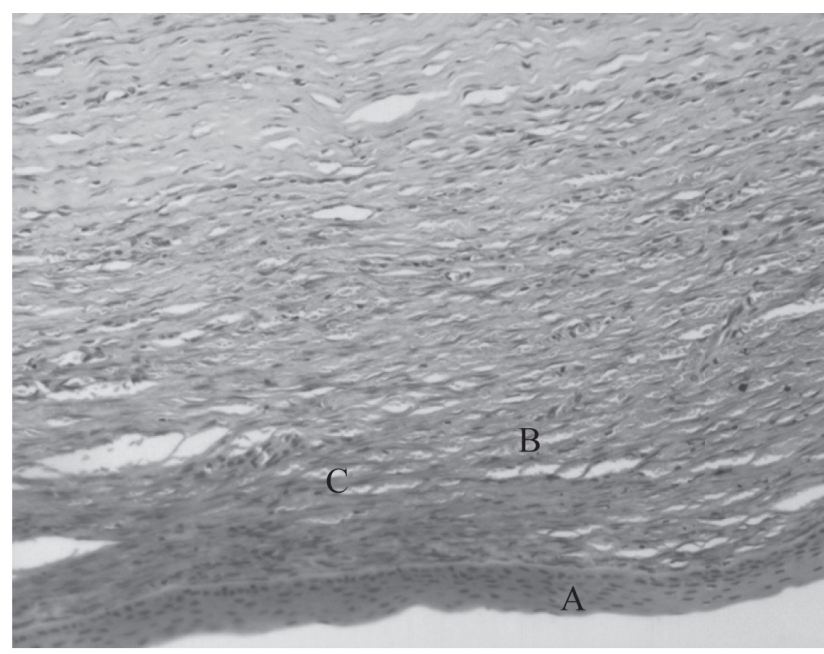

Figura 7 - Fotomicrografia de corte histológico de córnea com implante de MA equina, com 21 dias de evolução pós-operatória, mostrando a completa epitelização do implante (A).

Nota: a angiogênese é evidente neste período de observação (B), o implante já se encontra incorporado ao estroma e são observados feixes de fibras colágenas mais densamente distribuídos (C). Coloração: hematoxilina-eosina. Aumento: $100 \mathrm{x}$.

Fonte: (arquivos pessoais). 
densa na região da cicatriz e o infiltrado celular por fibrócitos e fibroblastos apresentava menor intensidade quando comparado ao observado no grupo de 21 dias (Figura 8). Nessa fase, a coloração PAS evidenciou a presença de lâmina basal epitelial reconstituída.

\section{Discussão}

Dentre os métodos de reparação da superfície ocular, a MA tem-se consolidado como útil e seu uso vem sendo amplamente pesquisado em medicina veterinária.

A glicerina $98 \%$ foi o método de escolha para manutenção da MA por ser simples e barato (MARAL et al., 1999), permitindo sua preservação por tempo ilimitado (BARROS et al., 1995). A glicerina tem ação antimicrobiana lenta (MARAL et al., 1999) e, portanto, a membrana só foi utilizada após um período mínimo de 30 dias de conservação (BARROS et al., 1995).

Após a ceratectomia, o segmento de MA foi aplicado com a face mesenquimal em contato com o estroma, em forma de retalho, pois, desta maneira, a MA é incorporada ao tecido receptor, funcionando como substrato para a proliferação das células epiteliais (AZUARA-BLANCO; PILLAI; DUA, 1999; DUA et al., 2004). O fio mononáilon 8.0 foi escolhido por seu baixo custo e fácil manipulação (GODOY; GUERRA; BARROS, 2002; SOUZA, 2003; TEIXEIRA; BARROS; MARTINS, 2004). Optou-se, neste estudo, pela retirada dos fios de sutura após a completa epitelização do implante, para evitar a resposta inflamatória adicional associada à presença dos mesmos (GODOY; GUERRA; BARROS, 2002; SOUZA, 2003).

Nas avaliações clínicas pós-operatórias, observouse que o blefarospasmo esteve presente em todos os cães e sua evolução foi semelhante àquela descrita por Souza (2003), de remissão total aos 15 dias de pósoperatório. Blefarospasmo, assim como fotofobia, foram observados em graus variados e com diferentes evoluções em ceratoplastias lamelares ou penetrantes com diversos implantes avaliados em trabalhos anteriores (GARCIA et al., 1996; TSENG; PRABHASAWAT; LEE, 1997; BARROS et al., 1998; ANDRADE

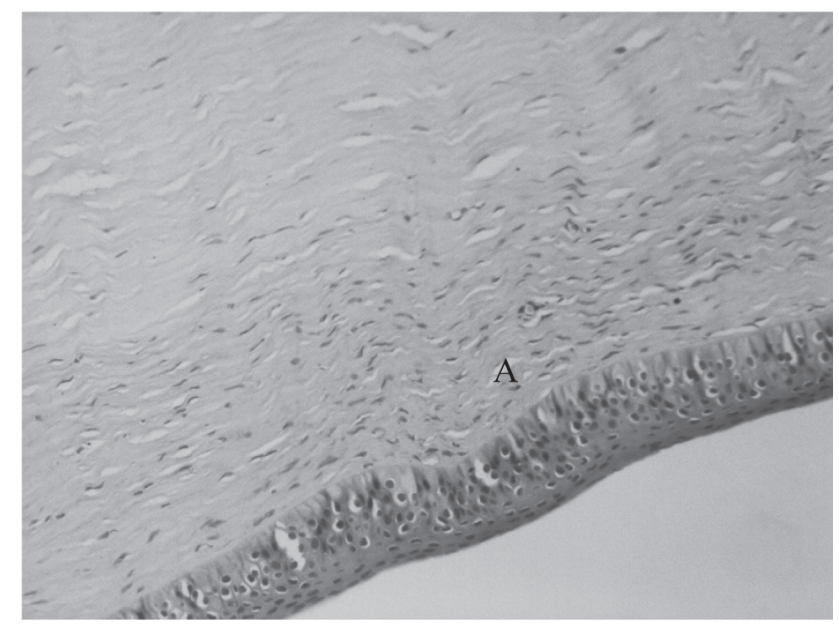

Figura 8 - Fotomicrografia de corte histológico de córnea com implante de MA equina, com 40 dias de evolução pós-operatória, mostrando tecido implantado incorporado, colagenizado e epitelizado (A). Coloração: hematoxilina-eosina. Aumento: $100 \mathrm{x}$.

Fonte: (arquivos pessoais).

et al., 1999; GODOY; GUERRA; BARROS, 2002; ANDRADE, 2003; SOUZA, 2003; TEIXEIRA; BARROS; MARTINS, 2004). O blefarospasmo inicial provavelmente caracterizou manifestação de dor pela própria intervenção cirúrgica, exposição de terminações nervosas da córnea (MARTIN, 2005), pelo estímulo do nervo palpebral ocasionado pela presença dos pontos de sutura e por uveíte reflexa (KERN, 1990).

A presença de secreção mucosa em todos os períodos de avaliação, assim como sua ausência após a retirada dos pontos, se deve ao estímulo inflamatório às células caliciformes da conjuntiva (GODOY; GUERRA; BARROS, 2002), associado ao procedimento cirúrgico e à presença dos pontos. O componente purulento apareceu em apenas um dos animais e resolveu-se rapidamente, provavelmente por ação do antibiótico, ou ainda, pelas atividades antimicrobiana e anti-inflamatória da MA (GOMES et al., 1999; SOUZA, 2003).

A quemose, presente nos primeiros dois a quatro dias, indicou a resposta inflamatória aguda após a intervenção cirúrgica (KERN, 1990). A hiperemia conjuntival, mais intensa nos primeiros dias, desapareceu somen- 
te após a retirada dos pontos, cuja presença estimula constantemente a resposta inflamatória local. Hiperemia e quemose foram também observadas por Garcia et al. (1996); Barros et al. (1997); Barros et al. (1998); Godoy, Guerra e Barros (2002); Andrade (2003); Souza (2003) e Teixeira, Barros e Martins (2004).

O edema de córnea não foi uma observação constante, pois se manifestou em sete animais em diferentes fases da cicatrização, e esteve restrito à periferia do implante. Esse fenômeno também foi descrito por Barros et al. (1997); Barros et al. (1998); Godoy, Guerra e Barros (2002) e Souza (2003); nas ceratectomias lamelares, a lesão epitelial permite a penetração de água no estroma, levando ao edema periférico, que tende à resolução com a epitelização do defeito, como observado neste estudo (GODOY; GUERRA; BARROS, 2002).

A evolução da epitelização do implante foi avaliada pelo teste de fluoresceína, e deu-se por volta do décimo dia. Em todos os animais, observou-se impregnação do implante pelo corante, já que existe afinidade entre ambos (ANDRADE, 2003). Com a aplicação da MA equina conservada em glicerina para reparação de lesões penetrantes em córnea de cão, Barros et al. (1998) observaram teste de fluoresceína negativo somente aos 30 dias de pós-operatório. Já Garcia et al. (1996) referiram teste de fluoresceína negativo na região do implante aos sete dias de pós-operatório, em ceratoplastias lamelares com implante de peritôneo homólogo em cão, semelhante ao observado neste estudo.

À análise histológica, observou-se epitelização do implante aos sete dias de pós-operatório, levando à aparente divergência entre os dados clínicos e microscópicos. Isso provavelmente ocorreu porque no sétimo dia apenas áreas restritas do implante apresentavam impregnação por fluoresceína, assim, provavelmente, os cortes histológicos analisados não envolveram estas pequenas áreas ainda não epitelizadas. Esses resultados são compatíveis com aqueles encontrados em experimentos anteriores, com relação ao tempo de epitelização do implante (GODOY; GUERRA; BARROS, 2002; SOUZA, 2003; GODOY, 2005).
A neovascularização e a formação de vasos em estruturas inicialmente avasculares, como a córnea, pode ocorrer por distúrbios inflamatórios, infecciosos, degenerativos ou traumáticos (CHANG et al., 2001). A neovascularização foi observada em todos os estudos que utilizaram membranas biológicas na córnea (GARCIA et al., 1996; BARROS et al., 1998; GODOY; GUERRA; BARROS, 2002; SOUZA, 2003; TEIXEIRA; BARROS; MARTINS, 2004). A evolução da neovascularização da córnea, observada neste estudo, foi semelhante aos resultados descritos por Godoy, Guerra e Barros (2002) e Souza (2003).

A evolução da epitelização do implante, observada na análise morfológica à microscopia óptica de luz, foi semelhante à descrita por Godoy, Guerra e Barros (2002); Souza (2003) e Godoy (2005). Aos 21 dias, a epitelização do implante estava completa. A região de reparação apresentou maior número de camadas celulares sem, contudo, restabelecer sua arquitetura normal, sendo o mesmo processo observado por Gris et al. (2002) e Godoy (2005). A formação de células epiteliais entre o estroma da córnea e a membrana amniótica, que ocorreu em um dos animais neste experimento, foi relatada anteriormente por Souza (2003); Teixeira, Barros e Martins (2004) e Godoy (2005). Esse fenômeno parece estar associado ao infiltrado de células epiteliais, sob a membrana, no momento da sutura.

Segundo Swank e Hosgood (1996), a regeneração da membrana basal pode levar semanas ou meses, estando a adesão do epitélio ao estroma prejudicada até este período. Neste estudo, aos 40 dias, verificou-se reconstituição completa da membrana basal.

No experimento de Souza (2003), com ceratoplastia lamelar em cães usando membrana amniótica e alantoaminiótica alógenas, a proliferação de fibroblastos iniciou-se aos 15 dias e perdurou até o final da avaliação aos 60 dias. Neste estudo, a proliferação de fibroblastos foi observada aos sete dias e tornou-se mais intensa aos 21 dias; aos 40 dias de pós-operatório já havia redução desta celularidade na região do im- 
plante. Godoy, Guerra e Barros (2002) observaram intensa proliferação celular, aos sete dias, e, aos 60 dias, a celularidade no implante já foi considerada baixa. Barros et al. (1998), utilizando MA equina em lesões penetrantes, relataram presença de fibroblastos persistentes até os 60 dias de pós-operatório, diferindo dos resultados encontrados neste estudo, aos 40 dias. Invasão do implante por fibroblastos também foi descrita por Gris et al. (2002), em córneas humanas com três e sete meses pós-implante de MA. A presença dos fibroblastos, formando novas fibras colágenas em permeio às fibras do implante (BROWN et al., 1995), resultou na maior densidade de fibras colágenas na região da cicatriz aos 21 e aos 40 dias de pós-operatório, observada neste estudo.

Os polimorfonucleares estavam presentes apenas nos bordos das lesões, aos dois dias. Aos sete dias, o infiltrado inflamatório foi maior, mas, ainda considerado discreto. Aos 21 e 40 dias de pós-operatório, essas células já não foram encontradas na região receptora do implante. A ausência de infiltrado inflamatório aos 15 dias foi descrita por Godoy, Guerra e Barros (2002) e por Barros et al. (1995); todavia, Sou-

\section{Referências}

ANDRADE, A. L. Efeitos da membrana amniótica humana sobre a cicatrização de córnea de coelhos submetidas a traumas mecânicos e químicos: aspectos clínicos, morfológicos e bioquímicos. 2003. 253 f. Tese Doutorado - Faculdade de Medicina de Botucatu, Universidade Estadual Paulista, Botucatu, 2003.

ANDRADE, A. L.; LAUS, J. L.; FIGUEIREDO, F.; BATISTA, C. $\mathrm{M}$. The use of preserved equine renal capsule to repair lamellar corneal lesions in normal dogs. Veterinary Ophthalmology, v. 2, n. 2, p. 79-82, 1999.

AZUARA-BLANCO, A.; PILlAI, C. T.; DUA, H. S. Amniotic membrane transplantation for ocular surface reconstruction. Brazilian Journal of Ophthalmology, v. 83, n. 4, p. 933-402, 1999.

BARROS, P. S. M.; SAFATLE, A. M. V.; RIGUEIRO, M. Uso do pericárdio de eqüino conservado em glicerina como enxerto penetrante da córnea de cães. Estudo experimental. Brazilian Journal of Veterinary Research and Animal Science, v. 34, n. 3, p. 138-141, 1997.

BARROS, P. S. M.; GARCIA, J. A.; LAUS, J. L.; FERREIRA, A. L.; SALLES GOMES, T. L. The use of xenologous amniotic membrane to repair canine corneal perforation created by penetranting keratectomy. Veterinary Ophthalmology, v. 1, n. 2/3, p. 119-123, 1998. za (2003) relatou maior número de células aos dois dias, com redução no sétimo dia de pós-operatório. A resposta inflamatória reduzida justifica-se pelas características inerentes à MA, que reduz a invasão por polimorfonucleares e acelera a apoptose dos mesmos (SWANK; HOSGOOD, 1996), reduz a expressão de citocinas pró-inflamatórias, contém proteínas antiinflamatórias e inibidores das proteinases HICK et al., 2005), além de ser um tecido imunoprivilegiado (SHIMAZAKI; YANG; TSUBOTA, 1997; GOMES; GUERRA; BARROS, 1999; KUBO et al., 2001).

\section{Conclusões}

Com base nos resultados obtidos, foi possível concluir que:

- A MA equina mostrou-se viável como implante em córnea de cão, após ceratectomia, sendo incorporada ao estroma e resultando em restabelecimento aceitável da transparência no local da lesão;

- A MA equina não ocasionou resposta de rejeição no tecido receptor.
BARROS, P. S. M.; SATAFLE, A. M. V.; MALERBA, T. A.; BURNIER, J. M. The surgical repair of the cornea of the dog using pericardium as a keratoprothesis. Brazilian Journal of Veterinary Research and Animal Science, v. 32, n. 4, p. 251-255, 1995.

BARROS, P. S. M.; SAFATLE, A. M. V.; GODOY, C. A.; SOUZA, M. S. B.; BARROS, L. F. M.; BROOKS, D. E. Amniotic membrane transplantation for the reconstruction of ocular surface in three cases. Veterinary Ophthalmology, v. 8, n. 3, p. 189-192, 2005.

BROWN, C. T.; APPLEBAUM, E.; BANWATT, R.; TRINKAUSRANDALL, V. Synthesis of stromal glycosaminoglycans in response to injury. Journal of Cellular Biochemistry, v. 59, n. 1, p. 57-68, 1995.

CHANG, J. H.; GABISON, E. E.; KATO, T.; AZAR, D. T. Corneal vascularization. Current Opinions in Ophthalmology, v. 12, p. 242-249, 2001.

DUA, H. S.; GOMES, J. A. P.; KING, A. J.; MAHARAJAN, V. S. The amniotic membrane in ophthalmology. Survey Ophthalmology, v. 49, n. 1 , p. $51-77,2004$.

GARCIA, J. A.; BARROS, P. S. M.; LAUS, J. L.; FERREIRA, A. L.; SAFATLE, A. M. V. Implante de peritônio homólogo conser- 
vado após ceratectomia lamelar em cães. Brazilian Journal of Veterinary Research and Animal Science, v. 33, p. 291-294, 1996. Supplement.

GODOY, C. A. L. Estudo comparativo da utilização de membranas amnióticas de coelha e humana como enxerto em ceratoplastia lamelar em coelhos. 2005. 94 f. Tese (Doutorado) Faculdade de Medicina Veterinária e Zootecnia, Universidade de São Paulo, São Paulo, 2005.

GODOY, C. A. L.; GUERRA, J. L.; BARROS, P. S. M. Cetatoplastia lamellar em cães utilizando membrana fetal equina como enxerto. Estudo experimental. Arquivos Brasileiros de Oftalmologia, v. 65 , n. 5, p. 545-549, 2002

GOMES, J. A. P.; KOMAGONE, C. M.; SANTOS, N.; CHAVES, A. P.; CUNHA, M. C.; FREITAS, D. Membrana amniótica nas cirurgias reconstrutivas da superfície ocular nas ceratoconjuntivites cicatriciais. Arquivos Brasileiros de Oftalmologia, v. 62, n. 5, p. 562-576, 1999.

GRIS, O.; WOLLEY-DOD, C.; GÜELL, J. L.; TRESSERRA, F.; LERMA, H.; CORCOSTEGUI, B.; ADÁN, A. Histologic findings after amniotic membrane graft in human cornea. Ophthalmology, v. 109, n. 3 , p. $508-512,2002$.

HICK, S.; DEMER, P. E.; BRUNETTE, I.; LA, C.; MABON, M.; DUCHESNE, B. Amniotic membrane transplantation and fibrin glue in the management of corneal ulcers and perforations: a review of 33 cases. Cornea, v. 24, n. 4, p. 369-377, 2005.

KERN, T. J. Ulcerative keratitis. Veterinary Clinics of North America Small Animal Practice, v. 20, n. 3, p. 643-666, 1990.

KUBO, M.; SONODA, Y.; MURAMATSU, R.; USUI, M. Imunogenicity of human amniotic membrane in experimental xenotransplantation. Investigative Ophthalmology and Visual Science, v. 42, n. 7, p. 1539-1546, 2001.

MARAL, T.; BORMAN, H.; ARSLAN, H.; DEMIRHAN, B.; AKINBINGOL, G.; HABERAL, M. Effectiveness of human amnion preserved long-term in glycerol as a temporary biological dressing. Burns, v. 25, n. 7, p. 625-635, 1999.

MARTIN, C. L. Cornea and sclera. In: Ophthalmic disease in veterinary medicine. London: Manson Publishing, 2005. p. 241-288.

MELLER, D.; PIRES, R. T.; MACK, R. J.; FIGUEIRREDO, F.; HEILIGENHAUS, A.; PARK, W. C.; PRABHASAWAT, P.; JOHN, T.; McLEOD, S. D.; STEUHL, K. P.; TSENG, S. C. Amniotic membrane transplantation for acute chemical or thermal burns. Ophthalmology, v. 107, n. 5, p. 980-990, 2000.

MILANI, J. F.; BARROS, P. S. M.; GUERRA, J. L.; BROOKS, D. Effects of topical $0.2 \%$ Cyclosporine A on corneal neovascularization induced by xenologous amniotic membrane implantation into a corneal stroma micropocket of rats. Pesquisa Veterinária Brasileira, v. 28, n. 8, p. 379-386, 2008.

PIRES, R. T. F.; PIRES, J. L. T. F.; TSENG, S. C. G. Transplante de membrana amniótica para reconstrução da superfície corneal e conjuntival. Arquivos Brasileiros de Oftalmologia, v. 62, n. 3, p. 340-346, 1999.

RÖTTH, A. de. Plastic repair of conjunctival defects with fetal membranes. Archives of Ophthalmology, v. 23, n. 3, p. 522-5, 1940.

SAFATLE, A. M. V.; BARROS, P. S. M.; MALUCELLI, B. E.; GUERRA, J. L. Implante de duas membranas biológicas em microbolsa corneana como modelo experimental de angiogênese. Brazilian Journal of Veterinary Research and Animal Science, v. 39, n. 4 , p. $189-195,2002$.

SHIMAZAKI, J.; YANG, H. Y.; TSUBOTA, K. Amniotic membrane transplantation for ocular surface reconstruction in patients with chemical and thermal burns. Ophthalmology, v. 104, n. 12, p. 2068-2076, 1997.

SLATTER, D. Cornea and sclera. In: Fundamentals of veterinary ophthalmology. 3. ed. Philadelphia: WB Saunders, 2001. p. 260-313.

SOUZA, M. S. B. Estudo comparativo entre a ceratoplastia lamelar em cães utilizando membranas amniótica e alantoamniótica alógenas preservadas em glicerina: avaliação clínica e morfológica. 2003. $161 \mathrm{f}$. Tese (Doutorado em Cirurgia) - Faculdade de Medicina Veterinária e Zootecnia, Universidade de São Paulo, São Paulo, 2003.

SWANK, A.; HOSGOOD, G. Corneal wound healing and the role of growth factors. Compendium on Continuing Education of Veterinary Practice, v. 18, n. 9, p. 1007-1014, 1996.

TEIXEIRA, R. B.; BARROS, P. S. M.; MARTINS, M. C. Effects of topical $2 \%$ cyclosporine A on the corneas of dogs subjected to lamellar keratoplasty with a graft of equine pericardium preserved in glycerin. Clinical and morphological evaluation. Ciência Rural, v. 34, n. 1, p. 131-138, 2004.

TSENG, S. C. G.; PRABHASAWAT, P.; LEE, S. H. Amniotic membrane transplantation for conjunctival surface reconstruction. American Journal of Ophthalmology, v. 124, n. 6, p. 765-74, 1997.

TSENG, S. C. G.; PRABHASAWAT, P.; BARTON, K.; GRAY, T.; MELLER, D. Amniotic membrane transplantation with or without limbal allografts for corneal surface reconstruction in patients with limbal stem cell deficiency. Archives of Ophthalmology, v. 116, p. 431-441, 1998. 